\title{
Les Samu-Smur en première ligne pour la prise en charge des arrêts cardiaques extrahospitaliers durant l'épidémie de Covid-19
}

\author{
French Pre-hospital Emergency Medical Services on the Front Line for Out-of-Hospital Cardiac \\ Arrests during the Covid-19 Epidemic
}

\author{
B. Vivien \\ Reçu le 28 octobre 2020 ; accepté le 30 octobre 2020 \\ (C) SFMU et Lavoisier SAS 2020
}

La pandémie de Covid-19 qui a atteint la France au $1^{\text {er }}$ semestre 2020, d'une ampleur au-delà de tout ce qui avait pu être imaginé auparavant, a conduit l'ensemble du système de santé, intrahospitalier et extrahospitalier, à mener des adaptations majeures, à marche forcée, non seulement en termes de stratégie et de filières de soins pour les régulations médicales des Samu, mais également sur le plan strictement médical en termes de protocoles de soins, tant au domicile par les équipes de Smur, que dans les services d'accueil des urgences [1-3].

Parmi les différentes pathologies auxquelles sont confrontées ces équipes, l'arrêt cardiaque extrahospitalier (ACEH) représente sans nul doute le challenge le plus important dans le contexte de cette épidémie de Covid-19 : urgence vitale extrême nécessitant l'intervention directe de plusieurs personnels sur un patient avec de multiples gestes urgents, exposant à un risque élevé de contamination, a fortiori lors de certains actes invasifs tels que l'intubation trachéale. À ce titre, Samu-Urgences de France (SUdF), la Société française de médecine d'urgence (SFMU) et le Conseil français de réanimation cardiopulmonaire (CFRC) ont émis des recommandations vis-à-vis de la prise en charge préhospitalière des arrêts cardiorespiratoires de patients adultes suspects ou confirmés d'infection par la Covid-19 [4,5]. Ces recommandations précisent en particulier les éléments permettant de réduire le risque d'exposition des personnels au risque de contamination : limitation du nombre d'intervenants en contact direct avec le patient, habillage systématique de chacun d'entre eux avec un équipement individuel de protection (EPI) préalablement à la prise en charge du patient, limitation du risque d'aérosolisation du virus durant la réanimation

B. Vivien $(\bowtie)$

Université de Paris et Samu de Paris,

hôpital universitaire Necker-Enfants Malades,

Assistance publique-Hôpitaux de Paris,

149, rue de Sèvres, F-75015 Paris, France

e-mail : benoit.vivien@aphp.fr cardiopulmonaire (RCP), ventilation manuelle avec un masque facial étanche et un filtre, intubation précoce et si possible à l'aide d'un vidéolaryngoscope. Élément important de ces recommandations, il est précisé que l'ensemble de ces consignes, visant à la protection des intervenants et à limiter leur risque de contamination, ainsi que le temps nécessaire à leur mise en œuvre, font partie intégrante du processus de prise en charge d'un patient en arrêt cardiaque dans le contexte d'un risque d'exposition à la Covid-19.

L'impact de la pandémie de Covid-19 sur la prise en charge des ACEH en Île-de-France a récemment été évalué par une équipe de l'Inserm [6]. Dans ce travail de registre, effectué sur la période allant du 16 mars au 26 avril 2020 représentant le pic de la première « vague » de cette épidémie, les auteurs ont rapporté un quasi-doublement de l'incidence des ACEH par comparaison avec les années 2012 à 2019 , avec en particulier une augmentation de près de $15 \%$ de l'incidence des ACEH survenant au domicile, cela étant probablement en rapport direct avec les mesures de confinement en vigueur à cette période. Cette étude a également relevé une diminution du taux de réanimation par les premiers témoins, de la fréquence des rythmes chocables, et surtout de la survie à l'admission hospitalière (odds ratio : $0,36$; IC $95 \%$ : $[0,24-0,52] ; p<0,001)$, toujours par comparaison avec les mêmes semaines des années précédentes. Alors que le délai moyen d'intervention des équipes préhospitalières n'était augmenté que d'une minute durant la période de l'étude (respectivement 10,4 vs 9,4 $\mathrm{min}, p<$ 0,001 ), les auteurs se sont pourtant hasardés à avancer une saturation des centres de réception et de régulation des appels (CRRA) des Samu comme étant l'un des éléments pouvant expliquer ce surcroît de mortalité.

Dans ce numéro des Annales françaises de médecine d'urgence, Gueugniaud et al. [7] ont évalué la prise en charge et le pronostic des ACEH selon le numéro d'appel de secours composé en première intention par les témoins, en l'occurrence le Samu (15) ou les sapeurs-pompiers (18). 
À partir des données du Registre national des arrêts cardiaques RéAC, les auteurs ont comparé les caractéristiques des ACEH survenus durant la période allant du $1^{\mathrm{er}}$ mars au 30 avril 2020 avec la même période des années 2012 à 2018. De manière similaire au travail précédent effectué par l'Inserm en Île-de-France, Gueugniaud et al. [7] ont constaté pour l'ensemble des ACEH au niveau national durant la période Covid une diminution de près de $50 \%$ du taux de retour d'une activité circulatoire spontanée (RACS), de la survie à l'admission, et de la survie à J30. Un élément particulièrement marquant est l'absence de survivant à $\mathrm{J} 30$ chez les patients suspectés ou confirmés d'une infection par la Covid-19 au moment de leur prise en charge, dont l'explication peut être multifactorielle : en premier lieu gravité de la pathologie sous-jacente (atteinte mixte cardiovasculaire et respiratoire, se traduisant par un délai de RACS plus prolongé), mais également retard à la mise en œuvre des premiers gestes par les témoins (entourage familial au domicile non formé) et RCP plus complexe (port d'EPI). Un deuxième résultat intéressant de ce travail est l'absence de différence de survie à tous les temps de l'analyse du pronostic selon le numéro d'appel initialement composé, 15 ou 18 , et ce quels que soient les sous-groupes de patients, ce qui va clairement à l'encontre de l'interprétation des auteurs de l'Inserm sur une perte de chance qui aurait été liée à la saturation des CRRA des Samu, versus les centres d'appels des sapeurs-pompiers. Enfin, lorsque l'appel initial était passé par le 15, les auteurs ont également constaté un délai d'arrivée plus long des sapeurs-pompiers, ainsi qu'une mise en œuvre moins fréquente de leur part de la RCP basique, versus un appel direct au 18. Le délai d'arrivée du Smur étant également plus rapide dans le cas d'un appel au 15 plutôt qu'au 18 (respectivement 18 vs $20 \mathrm{~min}, p<0,01$ ), le corollaire de ce résultat est une mise en œuvre plus précoce de la RCP médicalisée après un appel au 15 , avant même dans certains cas l'initiation par les sapeurs-pompiers de la RCP basique. Le pronostic de survie à tous les stades de l'analyse n'étant pas différent entre les appels au 15 ou au 18, il peut être supposé que le bénéfice du gain de temps pour la mise en œuvre de la RCP médicalisée après un appel au 15 soit contrebalancé par les caractéristiques des patients de ce groupe, plus âgés et plus souvent atteints de la Covid-19 comme l'ont identifié les auteurs.

Dans le contexte actuel où apparaissent ici et là des polémiques sur la gestion de la première « vague » Covid-19 en France par les Samu-Smur, ce travail de Gueugniaud et al. [7] apporte des éléments incontestables sur l'absence de perte de chance et de pronostic de survie des ACEH durant cette période selon que l'appel initial des témoins ait été passé au 15 ou au 18, et ce alors que la proportion des appels était relativement proche entre les deux services (respectivement 56 vs $44 \%$ ). Cela s'inscrit donc en faux vis-à-vis des partisans d'un numéro unique de secours, et la coexistence de plusieurs numéros d'appel doit être considérée a contrario comme une chance pour notre pays : le 15 pour les urgences médicales, ce que la population française a parfaitement intégré compte tenu de la recrudescence du nombre d'appels reçus par les CRRA pendant et depuis la crise Covid, et qui est également souligné dans le travail de Gueugniaud et al. [7] (proportion plus élevée d'ACEH d'origine médicale parmi les appels au 15), et le 18 pour les secours. A contrario, il est plus pertinent de s'intéresser à la dégradation globale du pronostic de survie des ACEH durant le pic de l'épidémie de Covid, mis en lumière tant par le travail de l'Inserm que par celui de Gueugniaud et al. [7], et ce quel que soit le numéro d'appel composé, 15 ou 18. La prochaine mise en œuvre du service d'accès au soin (SAS), en parallèle au déploiement national du nouveau système d'information SI-Samu, devrait permettre une harmonisation des outils d'interconnexion entre Samu et sapeurs-pompiers, et ce avec l'objectif final qui doit rester le nôtre et qui est simplement le bénéfice du juste soin pour le patient.

Liens d'intérêts : l'auteur déclare ne pas avoir de liens d'intérêts.

\section{Références}

1. Télion C, Marx JS, Dautreppe C, Carli P (2020) Retour d'expérience sur la régulation au Samu de Paris pendant la crise de Covid-19. Ann Fr Med Urgence 10:202-11

2. Morin F, Peschanski N, Ricard Hibon A, et al (2020) Retour d'expérience sur les transports Smur des patients Covid-19. Ann Fr Med Urgence 10:224-32

3. Drogrey M, Pernet J, Hausfater P (2020) Retour d'expérience sur la réorganisation d'un service d'urgence de centre hospitalouniversitaire en réponse à l'épidémie de Covid-19. Ann Fr Med Urgence 10:233-42

4. Groupe de travail de Samu-Urgences de France (SUdF) et du Conseil français de réanimation cardiopulmonaire (CFRC) 25 avril 2020. Recommandations concernant la protection des personnels et la prise en charge préhospitalière des arrêts cardiorespiratoires de patients adultes suspects ou confirmés d'infections à Covid19. https://www.cfrc.fr/documents/Recommandations\%20SUdFCFRC ACR\%20et\%20Covid 20200425.pdf?PHPSESSID=3eba3edd 629 cda4ed87d25361e040869 (Dernier accès le 29 octobre 2020)

5. Groupe de travail du Conseil français de réanimation cardiopulmonaire (CFRC), de la Société française de médecine d'urgence et de Samu-Urgences de France -26 mai 2020. Recommandations concernant la prise en charge des arrêts cardiaques de patients adultes par le grand public en période d'infections à Covid-19. https:// www.sfmu.org/upload/consensus/Texte\%20 citoyens $\% 20$ sauveteurs \%20covid\%202020-1.pdf (Dernier accès le 29 octobre 2020)

6. Marijon E, Karam N, Jost D, et al (2020) Out-of-hospital cardiac arrest during the Covid-19 pandemic in Paris, France: a populationbased, observational study. Lancet Public Health 5:e437-43

7. Gueugniaud PY, Baert V, Hugenschmitt D, et al (2020) Impact de la Covid-19 sur les arrêts cardiaques extrahospitaliers en phase de suractivité épidémique : données nationales du registre RéAC. Ann Fr Med Urgence 10:355-62 